\title{
Avaliação da intensidade da reação de Maillard, de atributos físico-químicos e análise de textura em doce de leite ${ }^{1}$
}

\author{
Júlia d'Almeida Francisquini ${ }^{2}$, Leandra Natália de Oliveira ${ }^{2}$, João Pablo Fortes Pereira ${ }^{2 *}$, Rodrigo Stephani ${ }^{3}$, \\ Ítalo Tuler Perrone ${ }^{4}$, Paulo Henrique Fonseca da Silva ${ }^{2}$
}

10.1590/0034-737X201663050001

\section{RESUMO}

Produzido e comercializado na América Latina, o doce de leite é muito apreciado por seus consumidores, com grande importância para o mercado brasileiro. A padronização das características desse produto, assim como sua adequação ao que é preconizado pelo Regulamento Técnico de Identidade e Qualidade, é dificultada pela grande diversidade cultural e variações tecnológicas da sua produção. Por essa razão, o estudo das características físico-químicas e reológicas do doce de leite torna-se de grande importância para se alcançar uniformidade, principalmente pela escassez de literatura técnica e científica sobre este produto. Neste trabalho, objetivou-se avaliar e relacionar os atributos físico-químicos e de textura. Foram analisadas doze marcas de doce de leite quanto aos teores de umidade, atividade de água e análise de textura, com posterior análise estatística dos dados. A análise estatística descritiva para os atributos de textura mostrou elevada variação entre as amostras. Quanto ao teor de umidade, 51,11\% das amostras tiveram resultados em desacordo com os padrões legais vigentes. As análises realizadas apresentaram resultados de HMF livre e total elevados. Pela Análise de Componentes Principais (PCA), verificou-se que a principal fonte de variação e de contribuição para agrupamento das marcas avaliadas está relacionada com os elementos que compõem as características reológicas do produto. Os resultados mostraram elevada correlação entre atividade de água, umidade e atributos reológicos. A combinação dos atributos físico-químicos e reológicos mostra-se útil para caracterização e avaliação da qualidade do doce de leite. Os resultados obtidos neste trabalho contribuem para ampliar os conhecimentos científicos e podem servir de subsídios para as indústrias de laticínios e a comunidade.

Palavras-chave: qualidade; reologia; atributos.

\section{ABSTRACT}

\section{Evaluation of Maillard Reaction intensity, Physical-chemistry Attributes and Texture Analysis in "Dulce de Leche"}

The Dulce de Leche, a product which is marketed and produced in Latin America, is much appreciated by consumers, with great importance for the Brazilian market. The standardization of the characteristics of this product as well as the suitability for recommendations of the Technical Identity and Quality Regulation becomes difficult due to the large cultural diversity and technological changes. In this context, the study of physical-chemical and rheological characteristics the Dulce de Leche becomes of great importance to achieve such uniformity, mainly by the lack of technical and scientific literature about this product. This work aimed to evaluate and relate the physical-chemical and texture attributes. Twelve Dulce de Leche brands were analyzed to moisture, water activity and texture analysis with

\footnotetext{
Submetido em 14/04/2015 e aprovado em 04/03/2016.

${ }^{1}$ Vinculado aos seguintes projetos: Especificações de produtos lácteos visando a certificação de laticínios - Fapemig 12.038; Doce de leite: indicadores de intensidade de tratamento térmico e implicações nutricionais - PROPESQ/UFJF.

${ }^{2}$ Universidade Federal de Juiz de Fora, Juiz de Fora, Minas Gerais, Brasil. jubisfrancisquini@gmail.com; leandra.oliveira@ufjf.edu.br; joao.pablo@ufjf.edu.br; paulo.henrique@ufjf.edu.br

${ }^{3}$ Gemacom Tech, Juiz de Fora, Minas Gerais, Brasil. rodrigo@gemacomtech.com

${ }^{4}$ Universidade Federal de Viçosa, Viçosa, Minas Gerais, Brasil. italo.perrone@ufv.br

*Autor correspondente: joao.pablo@ufjf.edu.br
} 
statistical analysis of the data. Descriptive statistical analysis for texture attributes showed high variability between samples. As for the moisture content, $51.11 \%$ of the samples were outside the statutory standards. The analysis had results of free HMF and elevated totals. Through the Principal Component Analysis (PCA) it was found that the main source of variation and contribution to group of the appraised brands is related to the elements that make up the rheological characteristics of the product. The results showed high correlation between water activity, moisture and rheological attributes. The combination of physical, chemical and rheological attributes were shown to be useful for characterization and evaluation of Dulce de Leche quality. These results expand scientific knowledge about Dulce de leche favoring the dairy industry and the scientific community.

Key words: quality; rheology; attributes.

\section{INTRODUÇÃO}

O doce de leite é citado nas referências internacionais como Dulce de leche, produzido e comercializado na América Latina, principalmente na Argentina e no Brasil. É apreciado pelos consumidores e obtido pela evaporação de leite acrescido de sacarose, adquirindo coloração, consistência e sabor característicos, em função de reações de escurecimento do tipo não enzimático (Demiate et al., 2001).

Para fabricação de doce de leite, parte-se de uma mistura de leite e sacarose, com, aproximadamente, $20 \mathrm{~kg}$ de sacarose para cada $100 \mathrm{~L}$ de leite, submetida ao calor, à pressão ambiente ou reduzida, até atingir cerca de 70\%, em massa, de sólidos totais (Tamime, 2009). Do ponto de vista físico-químico, é uma dispersão aquosa mista, composta principalmente por carboidratos (sacarose e lactose) e proteínas do leite (Ranalli et al., 2011). De acordo com Brasil (1997), "entende-se por Doce de Leite o produto, com ou sem adição de outras substâncias alimentícias, obtido por concentração e ação do calor a pressão normal ou reduzida do leite ou leite reconstituído, com ou sem adição de sólidos de origem láctea e /ou creme e adicionado de sacarose (parcialmente substituída ou não por monossacarídeos e/ou outros dissacarídeos)".

No Brasil, a produção de doce de leite é bastante diversificada, originando-se de fabricações artesanais a produções industriais em larga escala; sendo encontrado em todo o país. Essa diversidade implica, muitas vezes, falta de uniformidade e padronização. Percebe-se uma grande importância dos produtos lácteos, em especial do doce de leite, para o mercado nacional. No ano de 2012, a exportação desse produto atingiu cerca de 143 toneladas, ao valor de 445,6 milhões de dólares, enquanto a importação somou, aproximadamente, seis toneladas e um milhão de dólares (Milkpoint, 2013).

$\mathrm{O}$ conhecimento das propriedades físico-químicas e reológicas dos alimentos é necessário, uma vez que essas propriedades são úteis no controle de qualidade, design de processos, definição de transporte e condições de armazenamento. A determinação das características de textura dos alimentos em geral é de extrema importância, uma vez que essa propriedade tem a capacidade de afetar a percepção e aceitação dos consumidores (Rezaei et al., 2011). Conforme Bourne (2002), os principais fatores de qualidade dos alimentos são aqueles avaliados pela visão (cor, aparência, tamanho) e pelas cavidades bucal e nasal (sabor, odor). A textura é transmitida para o cérebro, por meio de sensores da boca, pela audição e pela memória, para construir uma "imagem" das propriedades de textura dos alimentos, compostas por seu conteúdo de proteínas, carboidratos, gorduras, sais minerais e vitaminas.

Verifica-se um déficit de estudos e de pesquisas, principalmente quanto à ocorrência de fraudes, além da existência de produtos fora de padrões, quanto à viscosidade, à cor e ao aroma. A produção industrial em larga escala do doce de leite impulsionou o surgimento de novas exigências com relação à qualidade, principalmente em termos de cor e de textura, além da necessidade do desenvolvimento e implantação de metodologias capazes de auxiliarem o controle e fiscalização por órgãos competentes (Silveira, 1995).

O tratamento térmico aplicado na fabricação do doce de leite sujeita esse à reações de escurecimento, as quais ocorrem não apenas durante o processamento, mas também ao longo do armazenamento, sendo um importante fator de determinação de sua qualidade (Chen et al., 2008).

De acordo com Shibao \& Bastos (2011), dentre as reações de escurecimento existentes, tem destaque a reação de Maillard, a qual tende a ocorrer em uma grande variedade de alimentos, como no doce de leite, causando importantes alterações de cor, sabor, valor nutricional, propriedades antioxidantes e textura do alimento. São fatores determinantes para o curso da reação o processamento a temperaturas acima de $40^{\circ} \mathrm{C}$, o pH entre 6 e 8 e a atividade de água de 0,4 a 0,7 . 
A reação de Maillard pode ser dividida em três fases. A inicial consiste na condensação da carbonila de um aminoácido e de um açúcar redutor, culminando na formação do primeiro composto estável da reação de Maillard, o produto de Amadori. Prolongando-se o aquecimento ou o armazenamento, inicia-se a segunda fase, caracterizada por reações químicas a partir dos compostos de Amadori, como desidratação, enolização e retroaldolização, gerando a formação de compostos dicarbonílicos, redutonas, derivados do furfural, como o 5-hidroximetilfurfural, além de produtos da degradação de Strecker. Esses produtos correspondem a compostos químicos com ampla variação da massa molar e ocorrem tanto nos alimentos submetidos a qualquer tipo de tratamento térmico, quanto no organismo humano, neste caso, denominada de reação de Glicação (Keeney \& Bassette, 1959; Shibao \& Bastos, 2011).

A última fase da reação de Maillard consiste na reação dos compostos intermediários com resíduos de lisina ou de arginina em proteínas, formando compostos estáveis, além de reações de fragmentação e polimerização, gerando melanoidinas e estruturas fluorescentes. Uma forma de reduzir o teor de produtos da reação de Maillard consiste na utilização de métodos mais brandos de cozimento e com alta atividade de água (Keeney \& Bassette, 1959).

O 5-hidroximetilfurfural (HMF) é um composto intermediário da reação de Maillard, detectável por técnica espectrofotométrica, que pode ser usado como indicador de tratamentos térmicos severos, como processamento UHT e esterilização (Mortier et al., 2000). Estudos recentes mostram a interferência desses produtos da reação de Maillard em processos biológicos, os quais estão relacionados com a inibição do crescimento celular, a redução da digestibilidade e a absorção de proteínas, a hipertrofia de órgãos, a redução de atividades enzimáticas e intestinais; além de possíveis perdas nutricionais por causa da reação de aminoácidos essenciais com o açúcar redutor presente no alimento (Shibao \& Bastos, 2011).

É possível a determinação de HMF livre e a de HMF total em alimentos. De acordo com Pinto \& WolfschonPombo (1984), considera-se HMF livre aquele determinado sem a presença de aquecimento, enquanto o HMF total corresponde àquelas substâncias formadas por resultado de aquecimento, reagindo com o ácido tiobarbitúrico. Silva (2004) descreve ainda HMF livre como sendo o representante do estádio de desenvolvimento da formação de HMF na ocasião da análise. Os valores de HMF total permitem estimar o quanto a reação de formação desse composto pode prosseguir.

Este trabalho teve por objetivos determinar as características físico-químicas e as texturas de amostras comerciais de doce de leite e quantificar seus índices de 5-hidroximetilfurfural (HMF), com a finalidade de se obter um melhor entendimento da concentração deste marcador no produto.

\section{MATERIAL E MÉTODOS}

Foram coletadas 12 marcas comerciais de doce de leite, as quais foram analisadas em duas repetições verdadeiras, totalizando 24 amostras verificadas. Cada análise foi executada em duplicata por amostra.

A determinação do teor de umidade foi realizada pelo método clássico gravimétrico, segundo Pereira et al. (2001), submetendo a amostra ao aquecimento em estufa, a $105^{\circ} \mathrm{C}$, por, aproximadamente, seis horas, até massa constante.

A análise de atividade de água foi conduzida no equipamento Aqua Lab 4 ATE e a análise de textura (gomosidade, elasticidade, dureza, adesividade, mastigabilidade e coesividade), com o analisador de Textura Brookfield CT3. A quantificação dos sólidos solúveis foi realizada por refratometria, em equipamento Reichert AR200.

A determinação de HMF livre e de HMF total foi conduzida por análise espectrofotométrica em meio acidificado, após desproteinização da amostra com ácido tricloroacético seguida de filtração e de adição de reagente cromogênico (ácido tiobarbitúrico), procedendo-se à leitura da absorvância em $443 \mathrm{~nm}$. A concentração do 5hidroximetilfurfural em $\mu \mathrm{mol} . \mathrm{g}^{-1}$ foi dada a partir da construção de uma curva analítica, utilizando-se diferentes níveis de padrão de 5-hidroximetilfurfural versus absorvância, segundo Keeney \& Bassette (1959).

Para a análise estatística descritiva dos dados, foi utilizado o software Microsoft Office Excel, 2007. A análise de correlação utilizou o mesmo software por meio do método de Pearson. Para aplicação do método multivariado de análise de componentes principais (Principal Components Analysis - PCA), utilizou-se o software MATLAB 7.5. Os dados obtidos para os atributos físico-químicos e reológicos foram normalizados, utilizando-se o préprocessamento SNV (Standard Normal Variate), o qual normaliza os dados usando a média ponderada. No modelo construído pela PCA, foram escolhidas duas componentes principais (CP's), com cerca de $98 \%$ da variância total capturada. A escolha das CP's foi baseada no gráfico de autovalores versus número de componentes principais, dado pelo software utilizado.

\section{RESULTADOS E DISCUSSÃO}

Os resultados da análise estatística descritiva para os atributos físico-químicos e reológicos de doce de leite, referentes a 24 amostras, estão apresentados na Tabela 1.

A baixa umidade do doce de leite pastoso melhora a conservação do produto, porém, facilita o aparecimento de arenosidade, defeito percebido sensorialmente (Demiate et al., 2001). Esse atributo mostrou-se alta variação entre as amostras analisadas, atingindo coeficiente de variação de $19,10 \%$. Disto depreende-se a falta de padronização 


\begin{tabular}{|c|c|c|c|c|c|c|c|c|c|c|c|}
\hline & $\begin{array}{l}\text { Umidade } \\
(\% \mathrm{~m} / \mathbf{m})\end{array}$ & $\begin{array}{c}\text { Sólidos } \\
\text { Solúveis } \\
\left({ }^{\circ} \text { Brix }\right)\end{array}$ & Aw & $\begin{array}{l}\text { HMF livre } \\
\left(\mu \mathrm{mol.g}{ }^{-1}\right)\end{array}$ & $\begin{array}{l}\text { HMF total } \\
\left(\mu \text { mol.g }^{-1}\right)\end{array}$ & $\begin{array}{c}\text { Gomosidade } \\
\text { (g) }\end{array}$ & $\begin{array}{c}\text { Elasticidade } \\
\quad(\mathbf{m J})\end{array}$ & $\begin{array}{c}\text { Dureza } \\
\text { (g) }\end{array}$ & $\begin{array}{c}\text { Adesividade } \\
\quad(\mathbf{m} \mathbf{J})\end{array}$ & $\underset{(\mathbf{m J})}{\text { Mastigabilidade }}$ & Coesividade \\
\hline \multicolumn{12}{|l|}{ Marca } \\
\hline $\mathrm{A}$ & 27,38 & 68,73 & 0,852 & 200,21 & 3385,40 & 513,60 & 10,35 & 563,00 & 24,96 & 52,13 & 0,84 \\
\hline B & 35,23 & 64,80 & 0,884 & 617,14 & 5461,59 & 584,50 & 11,57 & 723,50 & 22,85 & 66,32 & 0,70 \\
\hline $\mathrm{C}$ & 31,43 & 67,70 & 0,858 & 198,10 & 4684,87 & 438,30 & 14,48 & 445,00 & 25,35 & 62,24 & 0,93 \\
\hline $\mathrm{D}$ & 27,80 & 63,30 & 0,891 & 130,37 & 4344,13 & 454,20 & 18,54 & 546,00 & 37,88 & 82,58 & 0,80 \\
\hline E & 25,99 & 69,27 & 0,824 & 394,92 & 3577,99 & 561,60 & 19,35 & 643,50 & 58,74 & 106,56 & 0,84 \\
\hline F & 30,59 & 63,87 & 0,867 & 140,95 & 3567,41 & 656,70 & 17,99 & 922,50 & 46,13 & 115,86 & 0,63 \\
\hline G & 44,87 & 49,80 & 0,929 & 193,86 & 5103,92 & 341,80 & 9,94 & 674,00 & 6,48 & 33,31 & 0,44 \\
\hline $\mathrm{H}$ & 25,40 & 68,13 & 0,833 & 130,37 & 3946,24 & 1711,10 & 25,11 & 2569,50 & 260,44 & 421,36 & 0,49 \\
\hline I & 26,54 & 67,20 & 0,830 & 329,31 & 2680,63 & 1069,90 & 16,55 & 1682,00 & 121,46 & 173,64 & 0,50 \\
\hline $\mathrm{J}$ & 29,45 & 67,20 & 0,857 & 509,21 & 1838,31 & 404,80 & 14,07 & 547,50 & 47,89 & 55,85 & 0,71 \\
\hline K & 39,91 & 55,93 & 0,903 & 365,29 & 3728,25 & 235,50 & 18,60 & 268,50 & 18,72 & 42,95 & 0,84 \\
\hline $\mathrm{L}$ & 35,05 & 59,53 & 0,891 & 943,07 & 3509,21 & 464,00 & 17,03 & 598,50 & 39,46 & 77,48 & 0,62 \\
\hline Média Geral & 31,61 & 63,79 & 0,87 & 346,07 & 3818,99 & 619,67 & 16,13 & 1015,58 & 59,20 & 107,52 & 0,69 \\
\hline Valor máximo & 44,92 & 69,70 & 0,93 & 943,07 & 5461,59 & 1711,10 & 25,11 & 3469,00 & 260,44 & 421,36 & 0,93 \\
\hline Valor mínimo & 22,10 & 48,40 & 0,82 & 130,37 & 1838,31 & 235,50 & 9,94 & 281,50 & 6,48 & 33,31 & 0,44 \\
\hline Amplitude & 22,82 & 21,30 & 0,11 & 812,70 & 3623,28 & 1475,60 & 15,17 & 3187,50 & 253,96 & 388,05 & 0,49 \\
\hline Desvio padrão & 6,05 & 5,93 & 0,03 & 245,13 & 1001,06 & 400,75 & 4,34 & 900,35 & 69,79 & 106,01 & 0,16 \\
\hline Erro padrão da média & 1,19 & 0,99 & 0,006 & 70,76 & 288,98 & 115,69 & 1,25 & 259,91 & 20,15 & 30,60 & 0,05 \\
\hline Coeficiente de variação & $19,10 \%$ & $9,20 \%$ & $3,50 \%$ & $70,80 \%$ & $26,20 \%$ & $64,60 \%$ & $26,80 \%$ & $88,60 \%$ & $117,80 \%$ & $98,50 \%$ & $23,10 \%$ \\
\hline
\end{tabular}


entre os produtos colocados no mercado, o que se reflete em condição desfavorável para o consumidor, o qual pode encontrar alterações no consumo direto do doce de leite ou dificuldades no seu uso em preparações dietéticas.

A partir de análises de amostras de doce de leite, apresentadas por Demiate et al. (2001), percebe-se a heterogeneidade dos resultados de umidade, cujos valores oscilaram de 22,10 até 44,92\%.

Os atributos atividade de água e teor de sólidos solúveis apresentaram menores valores de coeficiente de variação, sendo úteis, igualmente, para verificação de condições de processamento e como critério de qualidade do doce de leite. Em estudo realizado por Milagres et al. (2010), o teor de sólidos solúveis para doce de leite comercial apresentou valor médio de $63^{\circ}$ Brix.

Ferreira et al. (2012), ao realizarem análise da atividade de água em seis marcas comerciais de doce de leite, encontraram valor médio de 0,88 ; semelhante ao deste estudo, para esse atributo (média de 0,86 ).

Embora o doce de leite não seja um produto favorável ao crescimento de micro-organismos, por apresentar alta concentração de carboidratos e, consequentemente, baixa atividade de água, há possibilidade de multiplicação de bolores e leveduras, não sendo excluído o desenvolvimento de bactérias patogênicas (Sá et al., 2012).

Para o teor de umidade, $51,11 \%$ dos resultados obtidos neste estudo estavam acima do limite estabelecido por Brasil (1997), correspondente a $30 \mathrm{~g} .100 \mathrm{~g}^{-1}$, o que mostra uma desconformidade com o regulamento em vigência para o produto em questão.

Em estudo semelhante, Demiate et al. (2001), ao analisarem 42 amostras de doce de leite comercializadas em São Paulo e Paraná, verificaram variações do teor de umidade de 19,0 a $37,2 \%(\mathrm{~m} / \mathrm{m})$, sendo $23,8 \%$ delas em desacordo com a legislação vigente.

O 5-hidroximetilfurfural (HMF) está presente em vários alimentos, como mel e doce de leite, e a presença de açúcares simples e de água em meio ácido fornece condições favoráveis para o aparecimento desse composto furânico. $\mathrm{O}$ HMF pode ser formado tanto nos alimento quanto nos organismos vivos, desempenhando atividade citotóxica, mutagênica e carcinogênica, o que pode destacar a importância do estudo dos compostos intermediários da reação de Maillard nos alimentos, que culmina em problemas nutricionais e para a saúde do ser humano (Silva et al., 2008).

Os valores de HMF nas amostras de doce de leite são variáveis de acordo com a composição do produto e se elevam linearmente com o aumento da concentração de açúcar e com o tempo de aquecimento da amostra tratada com ácido oxálico (Pinto \& Wolfschon-Pombo,1984).

Pinto \& Wolfschon-Pombo (1984) mostraram ainda a ocorrência de grande variabilidade dos valores de HMF livre e total, durante seus estudos com doce de leite, o que indica maior necessidade de cuidados na elaboração deste produto. Percebeu-se que doces de leite com menores teores de HMF livre e total ficaram nos primeiros lugares em um concurso de produtos lácteos, mediante avaliação por provadores treinados, realizado no VIII Congresso Nacional de Laticínios, concernentes aos atributos "características gerais" e "cor”, enquanto aqueles com maiores valores foram os últimos classificados no concurso.

As análises realizadas neste trabalho mostraram resultados de HMF livre e total bastante elevados, de 943,07 $\mu$ mol.g ${ }^{-1}$ e 5461,58 $\mu \mathrm{mol} . \mathrm{g}^{-1}$, respectivamente, sendo este último semelhante ao encontrado por Pinto \& WolfschonPombo (1984), ao analisarem 11 amostras de doce de leite apresentadas no Congresso Nacional de laticínios de 1984.

Para as amostras de doce de leite avaliadas, os resultados dos atributos de textura (gomosidade, elasticidade, dureza, adesividade, mastigabilidade e coesividade) permitem comprovar altas variações entre as amostras, implicando falta de padronização do produto. A utilização da análise reológica pelas indústrias de laticínios pode contribuir, decisivamente, para a padronização de produtos com características sensoriais desejáveis e esperadas pelos consumidores.

A Análise de Componentes Principais ( $P C A$ ) é um método exploratório capaz de separar as informações mais importantes, apresentando a finalidade básica de reduzir o dimensionamento dos dados, a partir de combinações lineares das variáveis originais. As observações podem então ser traçadas graficamente, facilitando a visualização e a avaliação de suas semelhanças e diferenças, determinando, dessa forma, se as amostras poderão ser ou não agrupadas (López-Camacho et al., 2013; Ringner, 2008; Soeiro, 2010).

Ao avaliar os resultados descritos na Figura 1 e os valores encontrados na Tabela 1 , verifica-se que a principal fonte de variação e de contribuição para agrupamento das marcas avaliadas está relacionada com os elementos que caracterizam a reologia do produto, ou seja, gomosidade, elasticidade, dureza, adesividade e mastigabilidade.

As marcas A, C, D, E, F e J, que representam 50\% do total de marcas analisadas, permaneceram agrupadas, visto que alcançaram valores muito semelhantes, tanto nos aspectos reológicos quanto nos físico-químicos.

O grupo formado pelas marcas B, K e L, apesar de elas apresentarem valores semelhantes para os aspectos reológicos, quando comparados com o grupo acima citado, mostrou valores superiores de atividade de água e de umidade e, consequentemente, valores inferiores para teor de sólidos solúveis. Este comportamento pode estar relacionado com o uso inadequado de ingredientes capazes de promover o aumento da viscosidade do produto por 
meio do aprisionamento de água livre do alimento, gerando manutenção das características sensoriais, ainda que o produto apresente baixo teor de sólidos solúveis. Ingredientes com a capacidade de modificar os requisitos sensoriais, principalmente quanto aos aspectos de textura dos alimentos, têm sido cada vez mais utilizados pela indústria, com o objetivo de obter produtos mais atrativos ao público consumidor. No entanto, esses ingredientes, muitas vezes, são utilizados em não conformidade com a legislação vigente, com o intuito de reduzir os custos e, consequentemente, aumentar a rentabilidade. $\mathrm{O}$ amido é um desses ingredientes que tem como principal função aprisionar a água livre presente no produto, levando ao aumento da viscosidade, ao controle da cristalização e à melhora dos aspectos de textura (Perrone et al., 2011).

Ao observar o comportamento da marca I, na Figura 1, e os valores mostrados pela Tabela 1, verifica-se que ela apresentou valores intermediários para todos os aspectos avaliados (físico-químicos e reológicos), não se enquadrando em um grupo específico.

Verifica-se ainda um contraste entre as marcas $\mathrm{G} \mathrm{e} \mathrm{H}$, evidenciado pela análise de PCA. Essa discrepância pode ser facilmente detectada, ao se analisar a Tabela 1 , em que a marca $\mathrm{H}$ apresenta os maiores valores para os aspectos reológicos de gomosidade, elasticidade, dureza, adesividade e mastigabilidade, todos estes influenciados pela possível presença de agentes espessantes. Já a marca G é caracterizada pelo baixo teor de sólidos solúveis e elevado teor de umidade, tendo, como consequência, menores valores para os aspectos reológicos avaliados.

Em decorrência da situação exposta, pode-se verificar uma possível falta de padronização das amostras analisadas. Percebe-se que as marcas que apresentaram resulta- dos mais discrepantes tiveram valores inferiores ou superiores aos da média da população, especialmente para os aspectos reológicos. Esses aspectos correlacionam-se com as características sensoriais e de textura do produto, o que aumenta ou reduz sua qualidade e aceitação pelo consumidor (Perrone et al., 2011).

Os resultados da análise de correlação são apresentados na Tabela 2. Os atributos atividade de água e umidade apresentaram correlação negativa $(-0,8567$ e $-0,8470$ respectivamente), com o teor de sólidos solúveis ( $\left.{ }^{\circ} \mathrm{Brix}\right), \mathrm{o}$ que pode ser atribuído à relação inversa entre teor de sólidos solúveis no alimento e sua quantidade de água. Houve ainda correlação negativa para umidade e atividade de água com os atributos gomosidade, elasticidade, dureza, adesividade, mastigabilidade e coesividade. Como exemplo desse fenômeno, verifica-se a associação inversa entre elevados índices de dureza, atributo que remete ao efeito de textura crocante do produto, o qual remete à resistência ou à deformação ou quebra do alimento dependendo dos teores de umidade e de atividade de água (Bourne, 2002).

HMF livre e HMF total também mostraram uma correlação negativa com teor de sólidos solúveis e todos os atributos reológicos. O HMF é um produto intermediário da reação de Maillard; sendo assim, seu teor no produto final está diretamente relacionado com a ocorrência dessa reação, que, por sua vez, pode ser controlada por parâmetros tecnológicos, como, por exemplo, o $\mathrm{pH}$ e a temperatura de aquecimento durante o processamento. Tem-se, ainda, que, durante o período de estocagem, com o aumento da viscosidade por adição de gomas, e consequente diminuição da cinética de reação, e água disponível, a ocorrência dessa reação também será modificada (Silva, 2004).

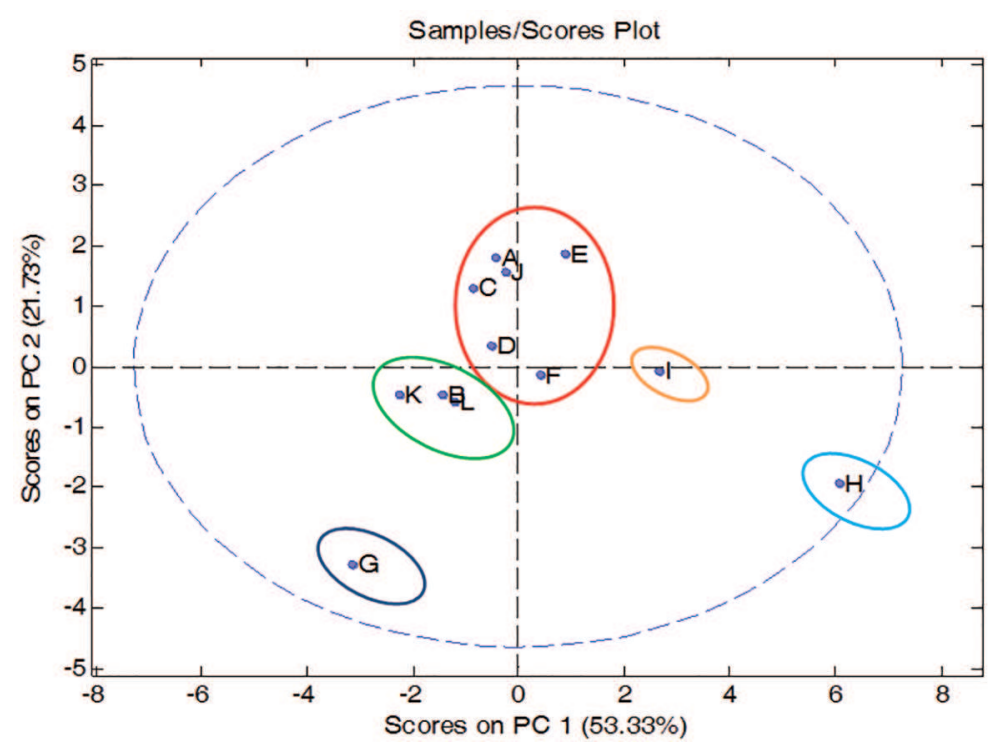

Figura 1: Gráfico de escores CP 1 versus CP 2 das amostras de doce de leite avaliadas considerando-se todos os atributos analisados. Condições: processo de normalização: SNV (Standard Normal Variate), número de componentes principais: 2, com aproximadamente $75 \%$ de variância captada. 


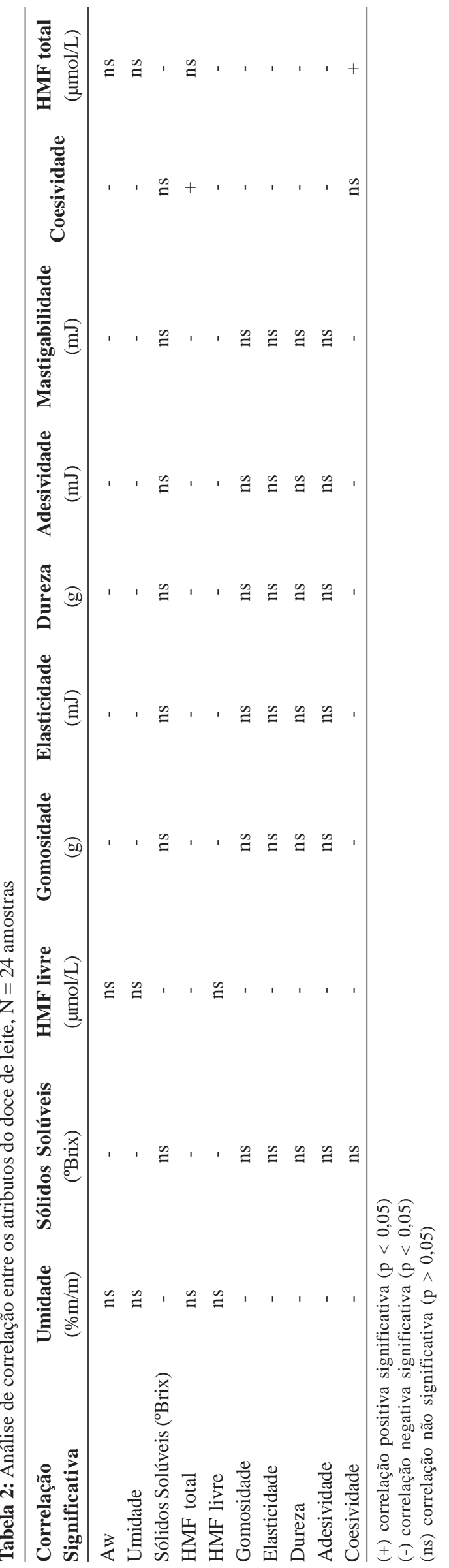

A indústria tem a liberdade tecnológica de definir o padrão de cor do seu produto final, de acordo com o público consumidor. Portanto, é de se esperar que para alguns produtos esta correlação seja válida e para outros não, visto que o desenvolvimento de coloração em doce de leite está intimamente relacionado com a ocorrência da reação de Maillard e produção de compostos de coloração escura (melanoidinas, produtos finais da reação) (Keeney \& Bassette, 1959; Shibao \& Bastos, 2011).

Os atributos reológicos entre si apresentaram correlações significativas enquanto os atributos físico-químicos que apresentaram melhor correlação com os demais foram atividade de água e umidade. Isso porque atividade de água e umidade estão intimamente relacionados com as características reológicas do produto bem como de ocorrência de reações.

\section{CONCLUSÃO}

Por este estudo depreende-se a relevância do conhecimento dos atributos físico-químicos e reológicos do doce de leite, produto amplamente consumido pela população brasileira. Verificou-se que os atributos físico-químicos, particularmente umidade e atividade de água, são capazes de apresentar correlações com as propriedades reológicas, as quais influenciam as características do produto final. Foi identificada falta de padronização de atributos físicoquímicos e reológicos entre as marcas analisadas. Neste estudo, mais da metade das amostras apresentaram resultados de umidade acima do limite estabelecido pela legislação vigente. Os procedimentos analíticos mostraram-se eficazes para a caracterização do doce de leite, sendo passíveis de adoção pelos órgãos regulamentadores e pelos fabricantes. Os resultados deste trabalho ampliam o conhecimento científico sobre doce de leite, favorecendo as indústrias de laticínios e a comunidade científica, buscando tanto a aderência aos requisitos legais quanto ao atendimento das expectativas dos consumidores no que concerne aos atributos de qualidade.

\section{REFERÊNCIAS}

Bourne M (2002) Food Texture and Viscosity: concept and measurement. 2 ${ }^{\mathrm{a}}$ ed. New York, Academic Press. 427p.

Brasil (1997) Ministério de Estado da Agricultura, Pecuária e Abastecimento. Portaria $n^{\circ} 354$, de 04 de setembro de 1997. Regulamento técnico de identidade e qualidade de doce de leite. DOU, 08/09/1997, Seção 1, p.19685.

López-Camacho E, Terashima-Marín H, Ochoa G \& ConantPablos SE (2013) Understanding the structure of bin packing problems through principal component analysis. International Journal of Production Economics, 145:488-499.

Chen SL, Yang DJ, Chen HY \& Liu SC (2009) Effect of hot acidic fructose solution on caramelisation intermediates including colour, hydroxymethylfurfural and antioxidative activity changes. Food Chemistry, 144:582-588. 
Demiate IM, Konkel FE \& Pedroso RA (2001) Avaliação da qualidade de amostras comerciais de doce de leite pastoso - composição química. Ciência e Tecnologia de Alimentos, 21:108-114.

Ferreira LO, Pereira PAP, Maria J \& Pinto SM (2012) Avaliação das características de qualidade de doces de leite comerciais. Revista do Instituto de Laticínios Cândido Tostes, 387:05-11.

Keeney M \& Bassete R (1959) Detection of intermediate compounds in the early stages of browning reaction in milk products. Journal of Dairy Science, Champaign, 42:945-961.

Milagres MP, Dias G, Magalhães MA, Silva MO \& Ramos AM (2010) Análise físico-química e sensorial de doce de leite produzido sem adição de sacarose. Revista Ceres, 57:439-445.

Milkpoint (2013) Balança Comercial de Lácteos de 2012. Disponível em: <http://www.milkpoint.com.br/cadeia-do-leite/ estatisticas/estatisticas-do-leite-milkpoint-80417n.aspx > Acessado em: 01 de agosto de 2013.

Mortier L, Braekman A, Cartuyvels D, Van Renterghem R \& De Block J (2000) Intrinsic indicators for monitoring heat damage of consumption milk. Biotechnology, Agronomy, Society and Environment, 4:221-225.

Pereira DBC, Silva PHF, Costa Junior LCG \& Oliveira LL (2001) Físico-química do leite e derivados - Métodos analíticos. $2^{\mathrm{a}}$ ed. Juiz de fora, Epamig. 234p.

Perrone IT, Stephani R \& Neves BS (2011) Doce de Leite: Aspectos Tecnológicos. Juiz de Fora, Do autor. 186p.

Pinto APEF \& Wolfschon-Pombo A (1984) 5-Hidroximetilfurfural no doce de leite. Revista do Instituto de Laticínios Cândido Tostes, 39:09-11.

Ranalli N, Andrés S \& Califano A (2012) Physicochemical and rheological characterization of "dulce de leche". Journal of Texture Studies, 43:115-123.
Rezaei R, Khomeiri M, Kashaninejad M \& Aalami M (2011) Effect of guar gum and arabic gum on the physicochemical, sensory and flow behaviour characteristics of frozen yoghurt. International Journal of Dairy Technology, 64:563-568.

Ringner M (2008) What is principal component analysis? Nature Biotechnology, 26:303-304.

Sá JFO, Perrone IT, Martins MF \& Silva PHF (2012) Qualidade microbiológica de doce de leite pastosos. Revista do Instituto de Laticínios Cândido Tostes, 386:61-66.

Shibao J \& Bastos DHM (2011) Produtos da reação de Maillard em alimentos: implicações para a saúde. Revista de Nutrição, 24:895-904.

Silva PHF (2004) Leite UHT: fatores determinantes para sedimentação e gelificação. Juiz de Fora, Templo. 124p.

Silva SJN, Schuch PZ, Vainstein MH \& Jablonski A (2008) Determinação do 5-Hidroximetilfurfural em méis utilizando cromatografia eletrocinética capilar micelar. Ciência e Tecnologia de Alimentos, 28:46-50.

Silveira NVV (1995) Grupo de trabalho: legislação de alimentos. Boletim da SBCTA, 29:77-78.

Soeiro BT, Boen TR, Pereira-Filho ER \& Lima-Pallone JA (2010) Investigação da qualidade de farinhas enriquecidas utilizando Análise por Componentes Principais (PCA). Ciência e Tecnologia de Alimentos, 30:618-624.

Tamime AY (2009) Dairy powders and concentrated products. Chennai, John Wiley \& Sons. 408p. 